\title{
Auditory hallucinations, not necessarily a hallmark of psychotic disorder
}

\author{
F. Waters ${ }^{1,2 *}$, J. D. Blom ${ }^{3,4,5}$, R. Jardri ${ }^{6}$, K. Hugdahl ${ }^{7,8}$ and I. E. C. Sommer ${ }^{9}$ \\ ${ }^{1}$ Clinical Research Centre, Graylands Health Campus, North Metropolitan Area Health Service Mental Health, Perth, Australia \\ ${ }^{2}$ School of Psychological Science, University of Western Australia, Perth, Australia \\ ${ }^{3}$ Faculty of Social Sciences, Leiden University, Leiden, the Netherlands \\ ${ }^{4}$ Parnassia Psychiatric Institute, The Hague, the Netherlands \\ ${ }^{5}$ Department of Psychiatry, University of Groningen, Groningen, the Netherlands \\ ${ }^{6}$ Univ Lille, CNRS UMR-9193, SCALab \& CHU Lille, Psychiatry Department, CURE Platform, Fontan Hospital, Lille, France \\ ${ }^{7}$ Division of Psychiatry, Haukeland University Hospital, Bergen, Norway \\ ${ }^{8}$ Department of Biological and Medical Psychology, University of Bergen, Bergen, Norway \\ ${ }^{9}$ Department of Neuroscience and Department of Psychiatry, University Medical Center Groningen, Groningen, the Netherlands
}

\begin{abstract}
Auditory hallucinations (AH) are often considered a sign of a psychotic disorder. This is promoted by the DSM-5 category of Other Specified Schizophrenia Spectrum And Other Psychotic Disorder (OSSSOPD), the diagnostic criteria for which are fulfilled with the sole presence of persistent AH, in the absence of any other psychotic symptoms. And yet, persistent $\mathrm{AH}$ are not synonymous with having a psychotic disorder, and should therefore not be uncritically treated as such. Many people who seek treatment for persistent AH have no other psychotic symptoms, have preserved realitytesting capacities, and will never develop a schizophrenia spectrum disorder. Instead, hallucinations may be the result of many different causes, including borderline personality disorder, post-traumatic stress disorder (PTSD), hearing loss, sleep disorders or brain lesions, and they may even occur outside the context of any demonstrable pathology. In such cases, the usage of the DSM-5 diagnosis of OSSSOPD would be incorrect, and it may prompt unwarranted treatment with antipsychotic medication. We therefore argue that a DSM-5 diagnosis of Schizophrenia Spectrum Disorder (or any other type of psychotic disorder) characterized by AH should require at least one more symptom listed under the A-criterion (i.e. delusions, disorganized speech, disorganized or catatonic behavior or negative symptoms). Adhering to these more stringent criteria may help to distinguish between individuals with persistent $\mathrm{AH}$ which are part of a psychotic disorder, for whom antipsychotic medication may be helpful, and individuals with $\mathrm{AH}$ in the absence of such a disorder who may benefit from other approaches (e.g. different pharmacological interventions, improving coping style, trauma-related therapy).
\end{abstract}

Received 14 March 2017; Revised 3 July 2017; Accepted 11 July 2017; First published online 22 August 2017

Key words: Diagnosis, DSM-5, hallucinations, psychosis, schizophrenia, voices.

\section{Introduction}

In the general community, and even among health professionals, the presence of auditory hallucinations (AH) is often considered synonymous with having a psychotic disorder (Parnas, 2013; Ford et al. 2014). This view is promoted by the diagnostic criteria for Other Specified Schizophrenia Spectrum and Other Psychotic Disorder (OSSSOPD), a diagnostic category first introduced in the fifth edition of the Diagnostic and Statistical Manual of Mental Disorders (DSM-5) (American Psychiatry Association, 2013). For related categories in the schizophrenia spectrum, such as Schizophrenia, Schizophreniform Disorder, and Schizoaffective

\footnotetext{
* Address for correspondence: F. Waters, Clinical Research Centre, c/o Graylands Hospital, Private Bag No 1, Claremont, WA 6910, USA. (Email: flavie.waters@health.wa.gov.au)
}

Disorder, at least two symptoms are required from those listed under the A-criterion (comprising delusions, hallucinations, disordered thinking, disorganized or catatonic behavior, and negative symptoms). To meet the DSM- 5 criteria for a diagnosis of OSSSOPD, however, the sole presence of persistent $\mathrm{AH}$ is sufficient. This is at odds with the fact that $\mathrm{AH}$ are experienced frequently in the context of other conditions, and sometimes even in the absence of any demonstrable pathology. Given that persistent $\mathrm{AH}$ are not always associated with a loss of contact with reality, lack of insight, or compromised dopaminergic transmission, it is questionable whether these types of $\mathrm{AH}$ should be treated with antipsychotic medication. Getting this issue right is also important given the stigma associated with a diagnosis of psychotic disorder and possible consequences for prognosis.

In this paper, we argue for a clearer distinction between $\mathrm{AH}$ as part of a psychotic disorder and those 
experienced in a different context, as the former may be an indication for antipsychotic drugs, while the latter may benefit from an alternative therapeutic approach. In what follows, we (i) consider the representation of hallucinations in the DSM-5 chapter on Schizophrenia Spectrum Disorders; (ii) highlight the crucial difference between psychotic disorders and psychotic symptoms; (iii) document alternative disorders with $\mathrm{AH}$ as a prominent feature which occur in relative isolation, i.e. in the absence of any other psychotic symptoms; (iv) discuss biological, environmental, and psychological explanations for AH that do not involve dopamine; and (v) consider whether AH are a risk factor for later psychosis. On the basis of these insights, we advocate a more stringent use of the DSM's A-criterion, requiring the presence of at least two symptoms in cases of OSSSOPD. Although the focus here is on nonverbal and verbal AH (i.e. 'voices'), our argument also applies to hallucinations experienced in other sensory modalities (i.e. visual, somatic, etc.).

\section{Hallucinations as a symptom domain in the DSM-5}

According to the DSM-5, Schizophrenia and Schizophreniform Disorder are diagnosed in the presence of two or more symptoms listed under the A-criterion, along with signs of persistence, distress, and impairments in functioning. One of the changes made after the DSM-IV was to drop the use of Schneiderian first-rank symptoms, including voices conversing and third-person hallucinations (Esquirol, 1845). Consequently, it is now possible to establish a diagnosis of Schizophrenia or Schizophreniform Disorder whenever hallucinations are present, regardless of form or content, as long as they are accompanied by a second symptom from the A-criterion, together with significant distress or impairment in functioning (APA, 2013).

The category OSSSOPD was devised for clinical presentations that are deemed 'characteristic' of the group of Schizophrenia Spectrum Disorders, but which do not meet all the diagnostic criteria of Schizophrenia or Schizophreniform Disorder. The diagnosis can be established in the sole presence of persistent $\mathrm{AH}$, i.e. in the absence of any other features (Box 1). An important benefit of this low-threshold residual category is that it helps to alert health professionals to the presence of $\mathrm{AH}$ in need of treatment, even in the absence of additional symptoms required for a diagnosis of Schizophrenia. Thus, patients suffering exclusively from distressing and burdensome hallucinations (for example command hallucinations) can be treated without delay. However, this creates a new problem of overinclusion, because any individual presenting with $\mathrm{AH}$ may now become labelled as having a psychotic disorder, even if the primary condition is a somatic disorder (e.g. migraine or
Box 1. DSM-5 criteria for Other Specified Schizophrenia Spectrum and Other Psychotic Disorder (OSSSOPD)

This category applies to presentations where are prominent symptoms characteristic of schizophrenia spectrum disorders that cause clinically significant distress or impairment in social, occupational, or other important areas of functioning but do not meet the full criteria for schizophrenia, schizophreniform disorder or schizoaffective disorder. The OSSSOPD category is used in situations in which the clinician chooses to communicate that the presentation does not meet the criteria for a more specific schizophrenia spectrum disorder, and allows him or her to provide a specific reason for this (e.g. 'persistent auditory hallucinations').

Examples of presentations that can be specified using the 'other specified' designation include the following:

(1) Persistent auditory hallucinations occurring in the absence of any other features.

(2) Delusions with significant overlapping mood episodes: This includes persistent delusions with periods of overlapping mood episodes that are present for a substantial portion of the delusional disturbance (such that the criterion stipulating only brief mood disturbance in delusional disorder is not met).

(3) Attenuated psychosis syndrome: This syndrome is characterized by psychotic-like symptoms that are below a threshold for full psychosis (e.g. the symptoms are less severe and more transient, and insight is relatively maintained).

(4) Delusional symptoms in partners of individual with delusional disorder: In the context of a relationship, the delusional material from the dominant partner provides content for delusional beliefs by an individual who may not otherwise entirely meet criteria for delusional disorder.

Parkinson's disease), other psychiatric disorder (e.g. dissociative disorder or personality disorder) or not demonstrable at all. The risk is that patients might be given unnecessary treatment and suffer from stigmatization. Incidentally, the risk of overinclusion is not unique to Schizophrenia Spectrum Disorders (Berrios, 1996), but this issue has received little attention in the literature.

\section{Psychotic disorder $v$. psychotic symptom}

Psychosis is a broad and rather non-specific term (David \& Ajnakina, 2016). It has been in use since the 1900s, when physicians began attributing mental disorders (or 'insanities') to underlying disorders of the brain. Since then, the term is featured in virtually every textbook of psychiatry, but is seldom explicitly defined. Even Sims (Sims, 2003), whose crystal-clear definitions of psychopathology have instructed generations of psychiatrists, has little more to say than that psychoses are 'exceedingly hard to define although they are usually said to be characterized by severe symptoms, such as delusions and hallucinations, and by lack of insight (...); there is loss of contact with reality.' In clinical practice, the term psychosis loosely encompasses a spectrum of diagnostic syndromes which includes schizophrenia and related disorders, the affective 
psychoses (i.e. major depressive disorder and bipolar disorder), substance-induced psychoses and withdrawal states, psychoses caused by a somatic disorder, and psychotic states associated with neurodegenerative disorders such as dementia, Parkinson's disease and Huntington's disease. Delirium is also sometimes included as a type of psychosis.

The term 'psychosis' therefore lacks a definition which provides meaning by specifying its necessary and sufficient conditions (i.e. an intensional definition). Attempts to provide such an intensional definition in terms of 'loss of contact with reality' or 'lack of insight' fail to cover the meaning of psychosis. After all, a loss of contact with reality cannot be considered sufficient for psychosis, as this feature may occur in other disorders such as autism, depressive disorder or eating disorder; nor is it a necessary feature, since individuals suffering from hallucinations and negative symptoms may still be in contact with reality. The same holds true for lack of insight (David, 1990; Varga et al. 2006).

Instead, 'psychosis' owes its meaning to extensional definitions which vary somewhat between authors, but usually consist of lists of psychotic symptoms (such as those mentioned under the A-criterion above). If we ask ourselves why symptoms from that disparate group are called psychotic, we find that this serves to indicate that we consider them severe, and that we suspect them to signal a loss of contact with reality and/or a lack of insight. Moreover, historically, it suggests that we consider them to have a neurobiological cause even though the cause or mechanism may elude us. That is as close as we can currently get to an intensional definition of psychosis.

The question to be answered now, is whether all patients with one or more psychotic symptoms (such as persistent, stressful AH) should be considered as having an underlying psychotic disorder.

\section{Persistent AH in non-psychotic disorders}

It is widely recognized that $\mathrm{AH}$ can accompany virtually all psychiatric disorders, including borderline personality disorder and dissociative identity disorder $(40 \%)$, unipolar depression and bipolar disorder $(45 \%)$, anxiety disorders $(14 \%)$, autism $(6 \%)$, and posttraumatic stress disorder (15\%) (Blom \& Sommer, 2010; Sommer \& Kahn, 2014). In addition, AH can be a symptom of disorders of the nervous system, including Parkinson's disease, stroke, and migraine (Vreeburg et al. 2016), tumors or lesions to the temporal lobe, brainstem or thalamus (Braun et al. 2003), sleep disorders (Fortuyn et al. 2009), or due to the effects of alcohol and drugs. Sensory disturbances due to conditions which affect the auditory pathways (e.g. hearing loss, auditory cortex dysfunction, etc.) are also a common cause.

Hallucinations in these different groups of clinical disorders can be persistent and distressing, and be associated with a depressed mood, anxiety, and suicidal behavior (Honig et al. 1998; Okulate \& Jones, 2003; Blom \& Sommer, 2010; Kingdon et al. 2010; Preti et al. 2014). Even though such AH can also present with significant impairments in social, occupational and other areas of functioning, they are not always accompanied by problems with reality testing, lack of insight or disturbed beliefs, and therefore cannot be considered as part of a psychotic disorder.

\section{Hallucinations in individuals in the general population}

One such example is that of $\mathrm{AH}$ reported by individuals in the general population. In the so-called 'extended phenotype' (van Os et al. 2009), prevalence estimates of hallucinations vary from $4 \%$ to $21 \%$ (Beavan et al. 2011). Hallucinations in this group are often transient and sporadic in nature (Hanssen et al. 2005), although $\mathrm{AH}$ can be recurrent and persistent in a minority (4\%) (McGrath et al. 2015). In this latter group, AH can be highly distressing in approximately 50\% of cases (Honig et al. 1998; Daalman et al. 2011; Woods et al. 2015), and may last for over a year (Peters et al. 2016). In one study of 13 voice hearers in the general population, hallucinations had been ongoing for longer than 4 years in $89 \%$ of cases (Leudar et al. 1997). These types of hallucination present mostly without any other psychotic symptoms, and there is a marked absence of psychopathology but for schizotypal traits (Sommer et al. 2010).

\section{Explanations for $\mathrm{AH}$ which do not involve dopamine}

Dopamine has once been hailed as the 'wind of the psychotic fire' (Laruelle \& Abi-Dargham, 1999), and antipsychotic medications with their antagonistic effects on dopamine receptors are routinely used to treat psychotic symptoms, whether or not in combination with psychosocial approaches. Evidence is accumulating, however, that the causes of $\mathrm{AH}$ do not always involve abnormal dopamine functions, raising questions about the systematic usage of antipsychotic medications for all symptoms considered as psychotic.

\section{Pharmacological evidence}

Dopaminergic overactivity in the striatum (Abi-Dargham et al. 1998) is the primary neuropharmacological explanation for positive symptoms in schizophrenia disorders. In support, Positron Emission Tomography (PET) studies using an F-Dopa tracer show that many 
individuals with these disorders have increased presynaptic striatal dopamine production (Howes et al. 2011). Using the same method, a study in individuals from the general population (e.g. nonpsychotic) with frequent $\mathrm{AH}$ failed to show any such increases of striatal dopamine production (Howes et al. 2013). This perhaps suggests that individuals who participated in the latter study may not have been benefited from antipsychotic treatment in the treatment of their hallucinations. In support, other PET studies indicate that targeting dopamine $\left(D_{2}\right)$ receptors with antipsychotic medication is only beneficial for individuals with increased striatal dopamine production, and not for those with normal production levels (Demjaha et al. 2012; Ćurčić-Blake et al. 2017).

Consequently, it is perhaps not surprising that recent evidence is pointing to neurotransmitter systems other than dopamine in the mediation of $\mathrm{AH}$ (Deamer \& Wilkinson, 2015). In regards to the glutamatergic system, for example, studies show elevated levels of glutamate and glutamine metabolites in the temporal and frontal brain regions of individuals diagnosed with schizophrenia with frequent and severe AH (Hayward et al. 2009; Hugdahl et al. 2015; Ćurčić-Blake et al. 2017). In addition, studies with ketamine show that drugs which alter glutamatergic neurotransmission are also capable of producing AH (Corlett et al. 2011). Such a role for the glutamatergic system cannot be linked directly to the dopamine hypothesis of psychotic disorders. Despite attempts to couple both views by considering a role for aberrant NMDA-R plasticity (Thomas et al. 2014), the glutamate system appears to form an alternative mediating pathway for $\mathrm{AH}$, consistent with the heterogeneity of conditions in which such hallucinations present.

To complicate things further, hallucinations in different sensory modalities - even when experienced in the context of the same disorder - may be mediated by different types of neurotransmitter. In the case of visual hallucinations, for example, 5HT-2a receptors appear to play a pivotal role given that serotonin agonists such as LSD and psilocybin can produce vivid hallucinations, which can subsequently be blocked with serotonin (5HT-2A) antagonists such as ketanserin and ritanserin (Vollenweider, 1998). This evidence points to different neurotransmitter pathways for hallucinations in different sensory modalities, again forcing the conclusion that the dopamine hypothesis of schizophrenia does not provide an explanation for all types of hallucination.

\section{Environmental and psychological explanations}

Other evidence that dopamine is not always directly involved in $\mathrm{AH}$ stems from studies on environmental and psychological factors. In vulnerable and older age groups, social isolation and withdrawal can be powerful causes for $\mathrm{AH}$ and other types of hallucination through the reduction of ordinary levels of sensory input. It has been speculated that the psychological effects of loneliness and social seclusion may prompt compensatory hypersensitivity of the perceptual network, with any hallucinations being projected outwards to meet the person's communicative needs (Hoffman, 2007). Psychological trauma is yet another potential cause for hallucinations. Some people can trace their symptom's onset back to traumatic events. This association has been found in many individuals diagnosed with schizophrenia who report abuse in childhood (Read et al. 2005), and to an even greater extent in individuals with borderline personality disorder and post-traumatic stress disorder experiencing AH. In the general community, traumatic events also have a substantive power to trigger hallucinations. For example, up to $80 \%$ of the recently bereaved report hallucinations of their loved one within the first month of the person's death (Grimby, 1993). Although predominantly visual in nature, these hallucinations can also have an auditory component. In youth, stressors such as bullying and sexual trauma are strong predictors of $\mathrm{AH}$ (Lardinois et al. 2011).

But even outside the context of trauma, psychological factors in general appear to play a pivotal role in prompting hallucinations. Hypervigilance, for example, and the way situations are appraised, act to shape and give credence to AH (Campbell \& Morrison, 2007), with anxiety acting to reduce the threshold for accepting ambiguous signals as real. Everyday examples include mothers believing their babies to be crying, and physicians on duty hearing the phantom ring of their pagers. In general, all conditions which are accompanied by high expectancy for a perceptual signal are a fertile ground for hallucinations.

Even though it is sometimes possible to treat such psychosocially-induced hallucinations with antipsychotics, psychosocial interventions should be the treatment of first choice. Options could include case-management approaches, psychoeducation, improving coping mechanisms, cognitive therapies (Hayward et al. 2009; Deamer \& Wilkinson, 2015), cognitive-behavioral therapy (CBT) (Thomas et al. 2014), trauma-related therapy (McCarthy-Jones \& Longden, 2015; Steel, 2015), voicedialoging (Corstens et al. 2012), mindfulness approaches (Strauss et al. 2015), and integrative treatments (Jenner et al. 2004).

In all, the above body of evidence converges with findings from the literature on schizophrenia, but also with the notion that there are many pathways independent of the dopamine system which are capable of eliciting hallucinations. 


\section{Are hallucinations a risk factor for later psychosis?}

The question of whether isolated $\mathrm{AH}$ constitute a risk factor for developing a psychotic disorder, and therefore whether individuals with these experiences should receive early therapeutic interventions, cannot be answered conclusively. At this stage, however, the existing evidence suggests that the risk is low, unless $\mathrm{AH}$ are accompanied by other psychotic symptoms and/or functional deficits. Longitudinal follow-up studies of individuals who first experienced $\mathrm{AH}$ in childhood or early adolescence confirm this (Poulton et al. 2000).

While the prevalence of hallucinations in children is relatively high (i.e. 9\%), these experiences are largely transient in nature (Jardri et al. 2014). A study of 337 children aged 7-8 years reporting hallucination-like experiences demonstrated that these experiences ceased spontaneously within 5 years in $76 \%$ of cases (Bartels-Velthuis et al. 2011). Rather than being associated with a later psychotic disorder, $\mathrm{AH}$ in children and adolescents are more frequently associated with a later depressive disorder, anxiety disorder or behavioral problems, even after adjusting for alcohol and illicit-substance abuse (Kelleher et al. 2012). Other studies concur, showing that the odds of $\mathrm{AH}$ alone to predict a psychotic disorder are low, unless they are accompanied by functional deficits or beliefs about malevolent intentions of the voices (Poulton et al. 2000; Daalman et al. 2016).

Other evidence comes from studies showing that the transition rate of young individuals with subclinical symptoms to psychosis varies widely. Psychotic symptoms that include hallucinations, delusional ideation and self-disturbances confer a relatively high risk for psychotic disorders in adulthood (Krabbendam et al. 2004), but less so when AH occur in isolation (Daalman et al. 2016). A number of variables appear to influence the transition to psychosis, including the persistence of symptoms over time, the onset of secondary delusions, depression, affective dysregulation and psychosocial dysfunction (Kaymaz \& van Os, 2010). Conduct disorder, when co-occurring with $\mathrm{AH}$, also predicts the transition to more severe forms of psychopathology (Askenazy et al. 2007).

Finally, studies of individuals in the prodromal phase (and in those with a diagnosis of schizophrenia spectrum disorder) show that no single symptom can predict the development of psychotic disorder or relapse into psychosis. More accurate predictions depend on features such as other psychotic manifestations, cognitive dysfunction, depression, and poor social functioning (Yung et al. 2003).

\section{Conclusions}

Altogether, $\mathrm{AH}$ and other hallucinations occur at relatively high rates in many different conditions and are not pathognomonic for any given disorder (including schizophrenia) (Ford et al. 2014). AH can occur without other psychotic symptoms and may be caused by a range of conditions in which dopaminergic transmission is not compromised. In all cases, an accurate diagnosis is critically important, as persistent $\mathrm{AH}$ as part of a psychotic disorder may be a good indication for antipsychotic medication, whereas persistent $\mathrm{AH}$ in the absence of such a disorder are probably not. Although antipsychotic medication can be very effective and potentially life-saving in individuals with psychotic disorders, it is questionable whether it should be offered to all individuals with $\mathrm{AH}$.

We believe the ambiguous use of the term 'psychosis' has caused much confusion in the minds of professionals and the lay public, and that the distinction between psychotic symptom and psychotic disorder is a particularly important one since psychotic symptoms can occur outside of a psychotic disorder. The habitual conceptualization of $\mathrm{AH}$ as a sure sign of schizophrenia spectrum disorder is a particularly good example of this problem, although a primary diagnosis of psychotic disorder is not justified for all individuals troubled by persistent hallucinations.

The roots of classification systems in conventional medical models require a diagnosis to rest on the presence and absence of several symptoms to determine whether the criteria for a specific syndrome or disorder may be fulfilled. In the traditional view of psychosis (as a state of distorted reality testing etcetera), the concomitant expression of at least one more psychotic symptom is critical. To that end, the DSM's A-criterion for schizophrenia is very useful, since it demands the presence of at least two psychotic symptoms. In the sole presence of persistent hallucinations, we argue that the diagnostic criteria for Other Specified Schizophrenia Spectrum And Other Psychotic Disorder is not helpful.

Alternative locations for the listing of hallucinations in textbooks of psychiatry include other syndromes and disorders where the presence of other symptoms fulfil the criteria for these conditions (e.g. PTSD, borderline personality disorder, etc). Where other symptoms or the underlying cause cannot be clearly established or ascertained, a broader category akin to the ICD's R44 ('symptoms involving general sensations and perceptions') may be helpful, perhaps under a new banner titled 'Perceptual disorders'. This new domain could include a class of perceptual phenomena causing distress and dysfunction, but without any impaired reality testing (including tinnitus, Charles Bonnet Syndrome, phantom limb pain, pareidolia, and Alice in Wonderland syndrome, to mention a few examples). This may help to improve differential diagnosis, and in differentiating between 
individuals who may benefit from antipsychotic medication and those who may not. Altogether, we should take note that the presence of persistent $\mathrm{AH}$ does not equate the presence of a psychotic disorder, and should therefore not uncritically be diagnosed or treated as such.

\section{Acknowledgement}

$\mathrm{KH}$ was funded by an ERC Advanced Grant \#693124, and a RCN grant \#223273.

\section{References}

Abi-Dargham A, Gil R, Krystal J, Baldwin RM, Seibyl JP, Bowers M, van Dyck CH, Charney DS, Innis RB, Laruelle M (1998). Increased striatal dopamine transmission in schizophrenia: confirmation in a second cohort. American Journal of Psychiatry 155, 761-767.

American Psychiatric Association (2013). Diagnostic and Statistical Manual of Mental Disorders, 5th edn. American Psychiatric Association: Washington.

Askenazy FL, Lestideau K, Meynadier A, Dor E, Myquel M, Lecrubier Y (2007). Auditory hallucinations in pre-pubertal children. A one-year follow-up, preliminary findings. European Child and Adolescent Psychiatry 16, 411-415.

Bartels-Velthuis AA, van de Willige G, Jenner JA, van Os J, Wiersma D (2011). Course of auditory vocal hallucinations in childhood: 5-year follow-up study. British Journal of Psychiatry 199, 296-302.

Beavan V, Read J, Cartwright C (2011). The prevalence of voice-hearers in the general population: a literature review. Journal of Mental Health 20, 281-292.

Berrios GE (1996). The History of Mental Symptoms: Descriptive Psychopathology Since the Nineteenth Century. Cambridge University Press: Cambridge, UK.

Blom JD, Sommer IEC (2010). Auditory hallucinations: nomenclature and classification. Cognitive and Behavioral Neurology 23, 55-62.

Braun CM, Dumont M, Duval J, Hamel-Hebert I, Godbout L (2003). Brain modules of hallucination: an analysis of multiple patients with brain lesions. Journal of Psychiatry $\mathcal{E}$ Neuroscience 28, 432-449.

Campbell ML, Morrison AP (2007). The relationship between bullying, psychotic-like experiences and appraisals in 1416-year olds. Behavioral Research \& Therapy 45, 1579-1591.

Corlett PR, Honey GD, Krystal JH, Fletcher PC (2011). Glutamatergic model psychoses: prediction error, learning, and inference. Neuropsychopharmacology 36, 294-315.

Corstens D, Longden E, May R (2012). Talking with voices: exploring what is expressed by the voices people hear. Psychosis 4, 95-104.

Ćurčić-Blake B, Bais L, Sibeijn-Kuiper A, Pijnenborg M, Knegtering H, Liemburg E, Aleman A (2017). Glutamate in dorsolateral prefrontal cortex and auditory verbal hallucinations in patients with schizophrenia: A $1 \mathrm{H}$ MRS study. Progress in Neuro-Psychopharmacology and Biological Psychiatry 78, 132-139.
Daalman K, Boks MP, Diederen KM, de Weijer AD, Blom JD, Kahn RS, Sommer IE (2011). The same or different? A phenomenological comparison of auditory verbal hallucinations in healthy and psychotic individuals. Journal of Clinical Psychiatry 72, 320-325.

Daalman K, Diederen K, Hoekema L, van Lutterveld R, Sommer I (2016). Five year follow-up of non-psychotic adults with frequent auditory verbal hallucinations: are they still healthy? Psychological Medicine 46, 1897-1907.

David AS (1990). Insight and psychosis. British Journal of Psychiatry 156, 798-808.

David AS, Ajnakina O (2016). Psychosis as a continuous phenotype in the general population: the thin line between normality and pathology. World Psychiatry 15, 129-130.

Deamer F, Wilkinson S (2015). The speaker behind the voice: therapeutic practice from the perspective of pragmatic theory. Frontiers in Psychology 6, 817.

Demjaha A, Murray RM, McGuire PK, Kapur S, Howes OD (2012). Dopamine synthesis capacity in patients with treatment-resistant schizophrenia. American Journal of Psychiatry 169, 1203-1210.

Esquirol E (1845). Mental Maladies; A Treatise on Insanity. Lea and Blanchard: Philadelphia, USA.

Ford JM, Morris SE, Hoffman RE, Sommer I, Waters F, McCarthy-Jones S, Thoma RJ, Turner JA, Keedy SK, Badcock JC, Cuthbert BN (2014). Studying hallucinations within the NIMH RDoC framework. Schizophrenia Bulletin 40(Suppl_4), S295-S304.

Fortuyn HAD, Lappenschaar G, Nienhuis FJ, Furer JW, Hodiamont PP, Rijnders CA, Lammers GJ, Renier WO, Buitelaar JK, Overeem S (2009). Psychotic symptoms in narcolepsy: phenomenology and a comparison with schizophrenia. General Hospital Psychiatry 31, 146-154.

Grimby A (1993). Bereavement among elderly people: grief reactions, post-bereavement hallucinations and quality of life. Acta Psychiatrica Scandinavica 87, 72-80.

Hanssen M, Bak M, Bijl R, Vollebergh W, van Os J (2005). The incidence and outcome of subclinical psychotic experiences in the general population. British Journal of Clinical Psychology 44, 181-191.

Hayward M, Overton J, Dorey T, Denney J (2009). Relating therapy for people who hear voices: a case series. Clinical Psychology \& Psychotherapy 16, 216-227.

Hoffman RE (2007). A social deafferentation hypothesis for induction of active schizophrenia. Schizophrenia Bulletin 33, 1066-1070.

Honig A, Romme MAJ, Ensink BJ, Escher SD, Pennings MHA, Devries MW (1998). Auditory hallucinations: a comparison between patients and nonpatients. Journal of Nervous and Mental Disease 186, 646-651.

Howes O, Bose S, Turkheimer F, Valli I, Egerton A, Stahl D, Valmaggia L, Allen P, Murray R, McGuire P (2011). Progressive increase in striatal dopamine synthesis capacity as patients develop psychosis: a PET study. Molecular Psychiatry 16, 885-886.

Howes OD, Shotbolt P, Bloomfield M, Daalman K, Demjaha A, Diederen KM, Ibrahim K, Kim E, McGuire P, Kahn RS, Sommer IE (2013). Dopaminergic function in the 
psychosis spectrum: an [18F]-DOPA imaging study in healthy individuals with auditory hallucinations.

Schizophrenia Bulletin 39, 807-814.

Hugdahl K, Craven AR, Nygård M, Løberg E-M, Berle JØ, Johnsen E, Kroken R, Specht K, Andreassen OA, Ersland L (2015). Glutamate as a mediating transmitter for auditory hallucinations in schizophrenia: A 1H MRS study.

Schizophrenia Research 161, 252-260.

Jardri R, Bartels-Velthuis AA, Debbané M, Jenner JA, Kelleher I, Dauvilliers Y, Plazzi G, Demeulemeester M, David CN, Rapoport J (2014). From phenomenology to neurophysiological understanding of hallucinations in children and adolescents. Schizophrenia Bulletin 40, S221-S232.

Jenner JA, Nienhuis FJ, Wiersma D, van de Willige G (2004). Hallucination focused integrative treatment: a randomized controlled trial. Schizophrenia Bulletin 30, 133.

Kaymaz N, van Os J (2010). Extended psychosis phenotype yes: single continuum - unlikely. Psychological Medicine 40, 1963-1966.

Kelleher I, Keeley H, Corcoran P, Lynch F, Fitzpatrick C, Devlin N, Molloy C, Roddy S, Clarke MC, Harley M, Arseneault L, Wasserman C, Carli V, Sarchiapone M, Hoven C, Wasserman D, Cannon M (2012).

Clinicopathological significance of psychotic experiences in non-psychotic young people: evidence from four population-based studies. British Journal of Psychiatry 201, 26-32.

Kingdon DG, Ashcroft K, Bhandari B, Gleeson S, Warikoo N, Symons M, Taylor L, Lucas E, Mahendra R, Ghosh S (2010). Schizophrenia and borderline personality disorder: similarities and differences in the experience of auditory hallucinations, paranoia, and childhood trauma. Journal of Nervous and Mental Disease 198, 399-403.

Krabbendam L, Myin-Germeys I, Hanssen M, Bijl RV, de Graaf R, Vollebergh W, Bak M, van Os J (2004).

Hallucinatory experiences and onset of psychotic disorder: evidence that the risk is mediated by delusion formation.

Acta Psychiatrica Scandinavica 110, 264-272.

Lardinois M, Lataster T, Mengelers R, Van Os J, Myin-Germeys I (2011). Childhood trauma and increased stress sensitivity in psychosis. Acta Psychiatrica Scandinavica 123, 28-35.

Laruelle M, Abi-Dargham A (1999). Dopamine as the wind of the psychotic fire: new evidence from brain imaging studies. Journal of Psychopharmacology 13, 358-371.

Leudar I, Thomas P, McNally D, Glinski A (1997). What voices can do with words: pragmatics of verbal hallucinations. Psychological Medicine 27, 885-898.

McCarthy-Jones S, Longden E (2015). Auditory verbal hallucinations in schizophrenia and post-traumatic stress disorder: common phenomenology, common cause, common interventions? Frontiers in Psychology 6, 1071 .

McGrath JJ, Saha S, Al-Hamzawi A, Alonso J, Bromet EJ, Bruffaerts R, Caldas-de-Almeida JM, Chiu WT, de Jonge P, Fayyad J, Florescu S (2015). Psychotic experiences in the general population: a cross-national analysis based on 31,261 respondents from 18 countries. JAMA Psychiatry 72, 697-705.
Okulate G, Jones O (2003). Auditory hallucinations in schizophrenic and affective disorder Nigerian patients: phenomenological comparison. Transcultural Psychiatry 40, 531-541.

Parnas J (2013). On psychosis: Karl Jaspers and beyond. In One Century of Karl Jaspers' General Psychopathology (ed. G. Stanghellini, T. Fuch), Chapter 14, pp. 208-226. Oxford University Press: Oxford, UK.

Peters E, Ward T, Jackson M, Morgan C, Charalambides M, McGuire P, Woodruff P, Jacobsen P, Chadwick P, Garety PA (2016). Clinical, socio-demographic and psychological characteristics in individuals with persistent psychotic experiences with and without a 'need for care'. World Psychiatry 15, 41-52.

Poulton R, Caspi A, Moffitt TE, Cannon M, Murray R, Harrington H (2000). Children's self-reported psychotic symptoms and adult schizophreniform disorder: a 15-year longitudinal study. Archives of General Psychiatry 57, 1053-1058.

Preti A, Sisti D, Rocchi MBL, Siddi S, Cella M, Masala C, Petretto DR, Carta MG (2014). Prevalence and dimensionality of hallucination-like experiences in young adults. Comprehensive psychiatry 55, 826-836.

Read J, van Os J, Morrison AP, Ross CA (2005). Childhood trauma, psychosis and schizophrenia: a literature review with theoretical and clinical implications. Acta Psychiatrica Scandinavica 112, 330-350.

Sims A (2003). Symptoms in the Mind: An Introduction to Descriptive Psychopathology. London: WB Saunders. Marrero, M.

Sommer I, Kahn RS (2014). Psychosis susceptibility syndrome: an alternative name for schizophrenia. Lancet Psychiatry 1, 111.

Sommer IE, Daalman K, Rietkerk T, Diederen KM, Bakker S, Wijkstra J, Boks MP (2010). Healthy individuals with auditory verbal hallucinations; who are they? Psychiatric assessments of a selected sample of 103 subjects. Schizophrenia Bulletin 36, 633-641.

Steel C (2015). Hallucinations as a trauma-based memory: implications for psychological interventions. Frontiers in Psychology 6, 1262.

Strauss C, Thomas N, Hayward M (2015). Can we respond mindfully to distressing voices? A systematic review of evidence for engagement, acceptability, effectiveness and mechanisms of change for mindfulness-based interventions for people distressed by hearing voices. Frontiers in Psychology 6, 1154.

Thomas N, Hayward M, Peters E, van der Gaag M, Bentall RP, Jenner J, Strauss C, Sommer IE, Johns LC, Varese F (2014). Psychological therapies for auditory hallucinations (voices): current status and key directions for future research. Schizophrenia Bulletin 40, S202-S212.

van Os J, Linscott RJ, Myin-Germeys I, Delespaul P, Krabbendam L (2009). A systematic review and meta-analysis of the psychosis continuum: evidence for a psychosis proneness-persistence-impairment model of psychotic disorder. Psychological Medicine 39, 179-195.

Varga M, Magnusson A, Flekkøy K, Rønneberg U, Opjordsmoen S (2006). Insight, symptoms and neurocognition in bipolar I patients. Journal of Affective Disorders 91, 1-9. 
Vollenweider FX (1998). Advances and pathophysiological models of hallucinogenic drug actions in humans: a preamble to schizophrenia research. Pharmacopsychiatry 31, 92-103.

Vreeburg S, Leijten F, Sommer I (2016). Auditory hallucinations preceding migraine, differentiation with epileptic origin: a case report. Schizophrenia Research 172, 222-223.
Woods A, Jones N, Alderson-Day B, Callard F, Fernyhough C (2015). Experiences of hearing voices: analysis of a novel phenomenological survey. Lancet Psychiatry 2, 323-331.

Yung AR, Phillips LJ, Yuen HP, Francey SM, McFarlane CA, Hallgren M, McGorry PD (2003). Psychosis prediction: 12-month follow up of a high-risk ('prodromal') group. Schizophrenia Research 60, 21-32. 